\title{
A REMARK ON UNIVERSAL COVERINGS OF HOLOMORPHIC FAMILIES OF RIEMANN SURFACES
}

\author{
Yoichi Imayoshi and MinORi Nishimura \\ Dedicated to the memory of Professor Nobuyuki Suita
}

\begin{abstract}
We study the universal covering space $\tilde{M}$ of a holomorphic family $(M, \pi, R)$ of Riemann surfaces over a Riemann surface $R$. The main result is that (1) $\tilde{M}$ is topologically equivalent to a two-dimensional cell, (2) $\tilde{M}$ is analytically equivalent to a bounded domain in $\mathbf{C}^{2}$, (3) $\tilde{M}$ is not analytically equivalent to the two-dimensional unit ball $\mathbf{B}_{2}$ under a certain condition, and (4) $\tilde{M}$ is analytically equivalent to the twodimensional polydisc $\Delta^{2}$ if and only if the homotopic monodoromy group of $(M, \pi, R)$ is finite.
\end{abstract}

\section{Introduction}

1.1. It is well-known as Koebe's uniformization theorem for a Riemann surface that the universal covering space $\tilde{R}$ of a complex manifold $R$ of dimension one is given as follows (cf. Bers [4] and Shafarevich [22], pp. 380401).

(1) $\tilde{R}$ is biholomorphically equivalent to the Riemann sphere $\hat{\mathbf{C}}$ if and only if $R$ is also biholomorphically equivalent to $\hat{\mathbf{C}}$.

(2) $\tilde{R}$ is biholomorphically equivalent to the complex plane $\mathbf{C}$ if and only if $R$ is biholomorphically equivalent to $\mathbf{C}, \mathbf{C} \backslash\{0\}$ or a torus.

(3) $\tilde{R}$ is biholomorphically equivalent to the unit disc $\Delta$ if and only if $R$ is not biholomorphically equivalent to $\hat{\mathbf{C}}, \mathbf{C}, \mathbf{C} \backslash\{0\}$ or a torus.

1.2. However, universal coverings and fundamental groups of complex manifolds of higher dimension are very complicated. We give some examples (cf. Shafarevich [22], pp. 401-408).

(1) There are infinitely many different simply-connected compact complex manifolds of dimension $n \geq 2$.

1991 Mathematics Subject Classification. Primary 32G15, Secondary 30F10, 30F60.

Key words and phrases. holomorphic family of Riemann surfaces, universal covering, uniformization, Teichmüller space, mapping class group.

Received March 9, 2004; revised August 17, 2004. 
(2) For a given finite group $\Gamma$, there exists a compact complex manifold of dimension $n \geq 2$ whose fundamental group is isomorphic to $\Gamma$.

(3) The polydisc $\Delta^{n}$ of dimension $n \geq 2$ is not biholomorphically equivalent to the unit ball $\mathbf{B}_{n}$ (Poincaré's theorem, cf. Narashimhan [17], p. 70).

1.3. P. A. Griffiths [8] got the following uniformization theorem of quasiprojective varieties. Here we describe the case of dimension two. Let $\hat{M}$ be a two-dimensional, irreducible, smooth quasi-projective algebraic variety over the complex number field. For every point $p$ in $\hat{M}$, there exists a Zariski neighborhood $M$ of $p$ such that $M$ has a holomorphic fibration $(M, \pi, R)$ of Riemann surfaces of type $(g, n)$ with $2 g-2+n>0$ over a hyperbolic Riemann surface $R$ of analytically finite type. (We give a definition of a holomorphic fibration in the next section.) Then Griffiths proved that the universal covering space $\tilde{M}$ is topologically equivalent to a two-dimensional cell and biholomorphically equivalent to a bounded domain of holomorphy in $\mathbf{C}^{2}$ by using the theory of simultaneous uniformization of Riemann surfaces due to Bers.

1.4. In this paper we study some function-theoretic properties of the universal covering space $\tilde{M}$ of a holomorphic family of Riemann surfaces $(M, \pi, R)$. Our Main results are follows:

THEOREM 1. The universal covering space $\tilde{M}$ of a holomorphic family of Riemann surfaces $(M, \pi, R)$ of type $(g, n)$ is not biholomorphically equivalent to the two-dimensional unit ball $\mathbf{B}_{2}$ provided that $(M, \pi, R)$ is locally trivial, $n>0$, or $R$ is not compact.

By Rosay's theorem [19] we have a corollary.

COROLlary 1. The universal covering space $\tilde{M}$ of a holomorphic family of Riemann surfaces $(M, \pi, R)$ of type $(g, n)$ is not biholomorphically equivalent to any two-dimensional strongly pseudoconvex domains provided that $(M, \pi, R)$ is locally trivial, $n>0$, or $R$ is not compact.

THEOREM 2. The universal covering space $\tilde{M}$ of a holomorphic family of Riemann surfaces $(M, \pi, R)$ is biholomorphically equivalent to the two-dimensional polydisc $\Delta^{2}$ if and only if all the fibers $S_{t}=\pi^{-1}(t)$ are biholomorphically equivalent.

As a corollary we have the following (see Imayoshi [9]).

COROLlary 2. The universal covering space $\tilde{M}$ of a holomorphic family of Riemann surfaces $(M, \pi, R)$ is biholomorphically equivalent to the two-dimensional polydisc $\Delta^{2}$ if and only if the homotopic monodromy group $\mathscr{M}$ of $(M, \pi, R)$ is finite.

In the case where $R$ has punctures, i.e., it is not compact, these results were obtained in Imayoshi [9]. In this paper we do not assume that $R$ has punctures. 
However, in Theorem 1 , if $R$ is compact, we assume that $(M, \pi, R)$ is locally trivial, or $n>0$, i.e., every fiber $S_{t}$ has punctures. It is known that a Kodaira surface $M$ has a locally non-trivial fibration $(M, \pi, R)$ of type $(g, 0)$ over a compact Riemann surface $R$ (Kas [12], Kodaira [14]), and its universal covering $\tilde{M}$ is not biholomorphically equivalent to $\mathbf{B}_{2}$ (Atiyah [1], Shabat [20], [21]). It is not known whether except for a kind of Kodaira surfaces there exits a locally non-trivial holomorphic family of Riemann surfaces of type $(g, 0)$ over a compact Riemann.

1.5. This paper is organized as follows: In $\S 2$ we give a definition of holomorphic families $(M, \pi, R)$ of Riemann surfaces and some examples of these families. In $\S 3$ we explain briefly Teichmüller theory used in this paper. In $\S 4$, using Teichmüller theory we construct canonically a universal covering space $\tilde{M}$ and its universal covering transformation group $\mathscr{G}$. Theorem 1 is proved in $\S 5$, and Theorem 2 is proved in $\S 6$ and $\S 7$.

\section{Holomorphic families of Riemann surfaces}

2.1. A holomorphic family $(M, \pi, R)$ of Riemann surfaces over a Riemann surface $R$ is defined as follows. Let $\hat{M}$ be a two-dimensional complex manifold, $C$ a one-dimensional analytic subset of $\hat{M}$ or an empty set, and $R$ be a Riemann surface. Assume that a proper holomorphic map $\hat{\pi}: \hat{M} \rightarrow R$ satisfies two conditions:

(i) by setting $M=\hat{M} \backslash C$ and $\pi=\hat{\pi} \mid M$, the holomorphic map $\pi$ is of maximal rank at every point of $M$, and

(ii) the fiber $S_{t}=\pi^{-1}(t)$ over each $t \in R$ is a Riemann surface of fixed analytically finite type $(g, n)$, where $g$ is the genus of $S_{t}$ and $n$ is the number of punctures of $S_{t}$, i.e., it is obtained by removing $n$ distinct points from a compact Riemann surface of genus $g$.

We call such a triple $(M, \pi, R)$ a holomorphic family of Riemann surfaces of type $(g, n)$ over $R$. We assume throughout this paper that $2 g-2+n>0$, and $R$ is a hyperbolic Riemann surface of analytically finite type.

2.2. We give some examples of holomorphic families of Riemann surfaces.

Example 1. Take two hyperbolic Riemann surfaces $R_{0}, S_{0}$ of analytically finite type. Let $M_{0}=R_{0} \times S_{0}$ and $\pi_{0}: M_{0}=R_{0} \times S_{0} \rightarrow R_{0}$ be the canonical projection. Then $\left(M_{0}, \pi_{0}, R_{0}\right)$ is a holomorphic family of Riemann surfaces of type $\left(g_{0}, n_{0}\right)$, where $\left(g_{0}, n_{0}\right)$ is the type of $S_{0}$.

A holomorphic family $(M, \pi, R)$ is said to be globally trivial if there exist biholomorphic maps $F: M \rightarrow M_{0}=R_{0} \times S_{0}$ and $f: R \rightarrow R_{0}$ with $\pi_{0} \circ F=$ $f \circ \pi$. A holomorphic family is said to be locally trivial if it is analytically a local tirivial fiber bundle. 
The universal covering $\tilde{M}_{0}$ of $M_{0}$ is biholomorphically equivalent to $\tilde{R}_{0} \times \tilde{S}_{0} \cong \Delta^{2}$. Poincaré's Theorem shows that $\tilde{M}_{0}$ is not biholomorphically equivalent to the unit ball $\mathbf{B}_{2}$. This is a trivial example of Theorems 1 and 2 .

Example 2. Let $R$ be a hyperbolic Riemann surface of analytically finite type $(g, n)$. Let $M=\{(p, q) \in R \times R \mid p \neq q\}$ and $\pi: M \rightarrow R$ be the canonical projection. Then $(M, \pi, R)$ is a locally non-tirivial holomorphic family of Riemann surfaces of type $(g, n+1)$. Theorems 1 and 2 imply that the universal covering $\tilde{M}$ of $M$ is biholomorphically equivalent to neither $\Delta^{2}$ nor $\mathbf{B}_{2}$.

Example 3. Set $R=\mathbf{C} \backslash\{0\}$ and $M=\left\{(x, y, t) \in \mathbf{C}^{2} \times R \mid y^{2}=x^{3}-t\right\}$. Let $\pi: M \rightarrow R$ be the canonical projection. Then $(M, \pi, R)$ is a holomorphic family of Riemann surfaces of type $(1,1)$, which is locally trivial, but not globally trivial. In this case $\tilde{M}$ is biholomorphically equivalent to $\Delta^{2}$.

Example 4. Set $\quad R=\mathbf{C} \backslash\{0,1\} \quad$ and $\quad M=\left\{(x, y, t) \in \mathbf{C}^{2} \times R \mid y^{2}=\right.$ $x(x-1)(x-t)\}$. Let $\pi: M \rightarrow R$ be the canonical projection. Then $(M, \pi, R)$ is a holomorphic family of Riemann surfaces of type $(1,1)$, which is not locally trivial. Hence Theorems 1 and 2 show that $\tilde{M}$ of $M$ is biholomorphically equivalent to neither $\Delta^{2}$ nor $\mathbf{B}_{2}$.

Example 5. Kodaira [14] constructed a locally non-trivial holomorphic family $(M, \pi, R)$ of Riemann surfaces of type $(g, 0)$ over a closed Riemann surface $R$. See also Atiyah [1], Barth, Peters and Van de Ven [2], Kas [12], and Riera [18]. We call such a complex surface $M$ a Kodaira surface.

Since this family is not locally trivial, Theorem 2 implies that $\tilde{M}$ is not biholomorphically equivalent to $\Delta^{2}$ (cf. Atiyah [1], p. 79). It is also known that $\tilde{M}$ is not biholomorphically equivalent to $\mathbf{B}_{2}$ (see Atiyah [1], p. 79).

Example 6. As stated in §1, for a two-dimensional, irreducible, smooth quasi-projective algebraic surface $\hat{M}$ over the complex number field and for every point $p \in \hat{M}$, there exists a Zariski neighborhood $M$ of $p$ such that $M$ has a holomorphic fibration $(M, \pi, R)$ of Riemann surfaces over a Riemann surface $R$.

\section{Teichmüller theory}

3.1. In order to construct canonically a universal covering space $\tilde{M}$ of a holomorphic family $(M, \pi, R)$ of Riemann surfaces of type $(g, n)$, we use Teichmüller theory. We shall explain it in brief (refer to Bers [5], and Imayoshi and Taniguchi [10]).

Let $S$ be a fixed Riemann surface of analytically finite type $(g, n)$ with $2 g-2+n>0$. A marked Riemann surface $\left(S, f, S^{\prime}\right)$ is a Riemann surface $S^{\prime}$ of analytically finite type $(g, n)$ with a quasiconformal map $f: S \rightarrow S^{\prime}$. We define 
an equivalence relation between marked surfaces $\left(S, f_{1}, S_{1}\right)$ and $\left(S, f_{2}, S_{2}\right)$ if there exists a conformal map $h: S_{1} \rightarrow S_{2}$ such that the self-map $f_{2}^{-1} \circ h \circ f_{1}: S \rightarrow S$ is homotopic to the identity. We denote by $\left[S, f, S^{\prime}\right]$ the equivalence class of a representative $\left(S, f, S^{\prime}\right)$. The Teichmüller space $T(S)$ of a Riemann surface $S$ is the set of all these equivalence classes $\left[S, f, S^{\prime}\right]$. Let $\operatorname{Mod}(S)$ be the set of all homotopy classes $\left[f_{0}\right]$ of quasiconformal self-maps $f_{0}: S \rightarrow S$. We call $\operatorname{Mod}(S)$ the Teichmüller modular group of $R$. Every element $\left[f_{0}\right]$ acts on $T(R)$ by

$$
\left[f_{0}\right]_{*}\left(\left[S, f, S^{\prime}\right]\right)=\left[S, f \circ f_{0}^{-1}, S^{\prime}\right] .
$$

3.2. Let $G$ be a finitely generated Fuchsian group of the first kind with no elliptic elements acting on the upper half-plane $U$ such that the quotient space $S \cong U / G$ is of type $(g, n)$. Let $Q_{\text {norm }}(G)$ be the set of all quasiconformal automorphisms $w$ of $U$ leaving $0,1, \infty$ fixed and satisfying $w G w^{-1} \subset \operatorname{PSL}(2, \mathbf{R})$, where $\operatorname{PSL}(2, \mathbf{R})$ is the set of all real Möbius transformations. Two elements $w_{1}$ and $w_{2}$ of $Q_{\text {norm }}(G)$ are equivalent if $w_{1}=w_{2}$ on the real axis $\mathbf{R}$. The Teichmüller space $T(G)$ of $G$ is the set of all equivalence classes $[w]$ obtained by classifying $Q_{\text {norm }}(G)$ by the above equivalence relation.

Let $L^{\infty}(U, G)_{1}$ be the complex Banach space of (equivalence classes of) bounded complex-valued measurable functions $\mu$ on $U$ satisfying

$$
\mu \circ g \frac{\overline{g^{\prime}}}{g^{\prime}}=\mu, \quad{ }^{\forall} g \in G, \quad \text { and } \quad\|\mu\|_{\infty}<1 .
$$

For an element $\mu \in L^{\infty}(U, G)_{1}$ denote by $w_{\mu}$ the element in $Q_{\text {norm }}(G)$ with Beltrami coefficient $\mu$. Let $W^{\mu}$ be the quasiconformal automorphism of the Riemann sphere $\hat{\mathbf{C}}$ such that $W^{\mu}$ has the Beltrami coefficient $\mu$ on the upper halfplane $U$, and comformal on the lower half-plane $L$, and

$$
W^{\mu}(z)=\frac{1}{z+i}+O(|z+i|)
$$

as $z \rightarrow-i$. This map $W^{\mu}$ is uniquely determined by $\left[w_{\mu}\right]$ up to the equivalence relation, i.e., $w_{\mu}=w_{v}$ on $\mathbf{R}$ if and only if $W^{\mu}=W^{v}$ on $L$. We set $T_{\beta}(G)=$ $\left\{\left[W^{\mu}\right] \mid \mu \in L^{\infty}(U, G)_{1}\right\}$, which is called the Bers Teichmüller space of $G$.

Let $\phi_{\mu}$ be the Schwarzian derivative of $W^{\mu}$ on $L$. Then $\phi_{\mu}$ is an element of the space $B_{2}(L, G)$ of bounded holomorphic quadratic differentials for $G$ on $L$. The space $B_{2}(L, G)$ is a $(3 g-3+n)$-dimensional complex vector space. Bers proved that the map sending $\left[W^{\mu}\right]$ into $\phi_{\mu}$ is a biholomorphic map of $T_{\beta}(G)$ onto a holomorphically convex bounded domain of $B_{2}(L, G)$, which is denoted the same notation $T_{\beta}(G)$.

Denote by $N(G)$ the set of all quasiconformal automorphisms $\omega$ of $U$ with $\omega G \omega^{-1}=G$. Two elements $\omega_{1}, \omega_{2} \in N(G)$ are equivalent if $\omega_{1}=\omega_{2} \circ g_{0}$ on the real axis $\mathbf{R}$ for some $g_{0} \in G$. Denote by $[\omega]$ the equivalence class of a representative $\omega$. Let $\operatorname{Mod}(G)$ be the set of all equivalence classes $[\omega]$ in $N(G)$. We call $\operatorname{Mod}(G)$ the Teichmüller modular group of $G$. Every element $[\omega]$ acts on $T(G)$ by 


$$
[\omega]_{*}([w])=\left[\lambda \circ w \circ \omega^{-1}\right]
$$

where $[w] \in T(G)$ and $\lambda \in P S L(2, \mathbf{R})$ with $\lambda \circ w \circ \omega^{-1} \in Q_{\text {norm }}(G)$.

\section{Construction of the universal covering space $\tilde{M}$ of a holomorphic family $(M, \pi, R)$ of Riemann surfaces}

4.1. We shall describe a way to construct a universal covering space $\tilde{M}$ of a given holomorphic family $(M, \pi, R)$ of Riemann surfaces of type $(g, n)$ by using Teichmüller theory. This is due to Griffiths [8].

Let $(M, \pi, R)$ be a holomorphic family of Riemann surfaces of type $(g, n)$ over $R$. Take a universal covering $\rho: \Delta \rightarrow R$ with covering transformation group $\Gamma$. Then there exists a holomorphic map $\Phi: \Delta \rightarrow T(S)$ sending $\tau \in \Delta$ into $\left[S, f_{\tau}, S_{\rho(\tau)}\right]$, where $f_{\tau}: S \rightarrow S_{\rho(\tau)}$ is a quasiconformal map moving continuously with resptect to the parameter $\tau$. We call this holomorphic map $\Phi: \Delta \rightarrow T(S)$ a representation of $(M, \pi, R)$ into a Teichmüller space $T(S)$. The representation $\Phi$ induces a group homomorphism $\Phi_{*}: \Gamma \rightarrow \operatorname{Mod}(S)$ satisfying $\Phi \circ \gamma=\Phi_{*}(\gamma) \circ \Phi$ for all $\gamma \in \Gamma$.

4.2. Identify $T(S)$ with $T_{\beta}(G)$. Then we obtain a representation $\Psi: \Delta \rightarrow$ $T_{\beta}(G)$ of $(M, \pi, R)$ into $T(G)$ and a biholomorphic map $F_{\tau}: D_{\tau} / G_{\tau} \rightarrow S_{\rho(\tau)}$ for each $\tau \in \Delta$, where $\Psi(\tau)=\left[W^{\mu(\tau)}\right], D_{\tau}=W^{\mu(\tau)}(U)$, and $G_{\tau}=W^{\mu(\tau)} G\left(W^{\mu(\tau)}\right)^{-1} \subset$ $\operatorname{PSL}(2, \mathbf{C})$.

We set

$$
\tilde{M}=\left\{(\tau, w) \mid \tau \in \Delta, w \in D_{\tau}\right\} .
$$

This set $\tilde{M}$ is topologically equivalent to a two-dimensional cell. From (3.1) Koebe's one-quarter theorem shows that $D_{\tau} \subset\{|w|<2\}$ for all $\tau \in \Delta$, and so $\tilde{M}$ is a bounded domain in $\mathbf{C}^{2}$. It is also shown that $\tilde{M}$ is a domain of holomorphy. Let $\tilde{\pi}: \tilde{M} \rightarrow \Delta$ be the holomorphic map sending $(\tau, w)$ into $\tau$. Then the fiber $\tilde{\pi}^{-1}(\tau)$ of $(\tilde{M}, \tilde{\pi}, \Delta)$ over $\tau$ is biholomorphically equivalent $D_{\tau}$.

Let $\Pi: \tilde{M} \rightarrow M$ be the holomorphic map sending $(\tau, w)$ into $F_{\tau}(w)$. Then $\Pi: \tilde{M} \rightarrow M$ is the universal covering of $M$ constructed by Griffiths [8].

4.3. We shall explicitly express the elements of the covering transformation group $\mathscr{G}$ of the the universal covering $\Pi: \tilde{M} \rightarrow M$. For each element $\gamma \in \Gamma$, the homotopic monodromy $\mathscr{M}_{\gamma}$ of $\gamma$ for $(M, \pi, R)$ is the element of the Teichmüller modular $\operatorname{group} \operatorname{Mod}(G)$ with $\Phi \circ \gamma=\mathscr{M}_{\gamma} \circ \Phi$. The subgroup $\mathscr{M}=\left\{\mathscr{M}_{\gamma} \mid \gamma \in \Gamma\right\}$ of $\operatorname{Mod}(G)$ is called the homotopic monodromy group of $(M, \pi, R)$ with respect to the representation $\Phi$.

Denote by $N(G)$ the set of all quasiconformal automorphisms $\omega$ of $U$ with $\omega G \omega^{-1}=G$. Take an element $\omega_{\gamma} \in N(G)$ inducing $\mathscr{M}_{\gamma}$, i.e., $\left[\omega_{\gamma}\right]=\mathscr{M}_{\gamma}$. We may assume that $\omega_{\gamma \circ \delta}=\omega_{\gamma} \circ \omega_{\delta}$ for all $\gamma, \delta \in \Gamma$.

For each $\tau \in \Delta$, let $\left[w_{\mu(\tau)}\right]$ be the point of $T(G)$ with Beltrami coefficient $\mu(\tau) \in L^{\infty}(U, G)_{1}$ corresponding to the $\Psi(\tau) \in T_{\beta}(G)$. For every $g \in G$, we set 
$w_{v(\tau)}=\lambda \circ w_{\mu(\tau)} \circ\left(\omega_{\gamma} \circ g\right)^{-1} \in Q_{\text {norm }}(G)$, where $\lambda$ is a real Möbius transformation. Note that $w_{v(\tau)}=w_{\mu(\gamma(\tau))}$.

If we set

$$
(\gamma, g)(\tau, w)=\left(\gamma(\tau), W^{\mu(\gamma(\tau))} \circ\left(\omega_{\gamma} \circ g\right) \circ\left(W^{\mu(\tau)}\right)^{-1}(w)\right),
$$

then the map $(\gamma, g)$ is an analytic automorphism of $\tilde{M}$ (see Bers [3], Theorem 2, p. 95). We set

$$
H(\gamma, g)(\tau, w)=W^{\mu(\gamma(\tau))} \circ\left(\omega_{\gamma} \circ g\right) \circ\left(W^{\mu(\tau)}\right)^{-1}(w) .
$$

Then $H(\gamma, g)(\tau, \cdot): D_{\tau} \rightarrow D_{\gamma(\tau)}$ is a conformal map such that $G_{\gamma(\tau)}=$ $H(\gamma, g)(\tau, \cdot) G_{\tau}(H(\gamma, g)(\tau, \cdot))^{-1}$ and $H(\gamma, g)(\tau, \cdot)$ induces a conformal map of $D_{\tau} / G_{\tau}$ onto $D_{\gamma(\tau)} / G_{\gamma(\tau)}$.

Now the covering transformation group $\mathscr{G}$ of the universal covering $\Pi: \tilde{M} \rightarrow M$ is identified with the set $\Gamma \times G$. By definition, we have the relation

$$
(\gamma, g) \circ(\delta, h)=\left(\gamma \circ \delta, \omega_{\delta}^{-1} \circ g \circ \omega_{\delta} \circ h\right)
$$

for all $\gamma, \delta \in \Gamma$ and $g, h \in G$, which implies that $\mathscr{G}$ is a semi-direct product of $\Gamma$ by $G$. Note that $(\gamma, g)=(\delta, h)$ if and only if $\gamma=\delta$ and $g=h$.

\section{Proof of Theorem 1}

5.1. In this section we shall give a proof of Theorem 1. We use the notation in $\S 3$ and $\S 4$.

If $(M, \pi, R)$ is locally trivial, then the representation $\Psi$ of $(M, \pi, R)$ into a Teichmüller space $T(G)$ is constant. Hence $\tilde{M}=\Delta \times D_{0} \cong \Delta \times \Delta$, which implies that $\tilde{M}$ is not biholomorphically equivalent to the unit ball $\mathbf{B}_{2}$ by Poincaré's Theorem.

If the base surface $R$ is not compact, the assertion of Theorem 1 is shown in Imayoshi [9], pp. 584-586.

5.2. Let us consider the case $n>0$, i.e., every fiber $S_{t}=\pi^{-1}(t)$ is not compact. Assume that there exists a biholomorphic map $F=\left(F_{1}, F_{2}\right): \tilde{M} \rightarrow \mathbf{B}_{2}$.

We may assume that for every $\Phi_{*}(\gamma)=\left[f_{\gamma}\right] \in \operatorname{Mod}(S), \gamma \in \Gamma$, the quasiconformal self-map $f_{\gamma}: S \rightarrow S$ fixes each puncture of $S$. In fact, the subgroup $\mathscr{M}^{\prime}=\left\{\left[f_{\gamma}\right] \in \Phi_{*}(\Gamma) \mid f_{\gamma}\right.$ fixes every puncture of $\left.S\right\}$ of $\Phi_{*}(\Gamma)$ is a normal subgroup $\mathscr{M}$ of finite index. Let $\Gamma^{\prime}=\left\{\gamma \in \Gamma \mid\left[f_{\gamma}\right] \in \mathscr{M}^{\prime}\right\}$. Then $\Gamma^{\prime}$ is a normal subgroup of $\Gamma$ and $\Gamma / \Gamma^{\prime}$ is canonically isomorphic to $\mathscr{M} / \mathscr{M}^{\prime}$. Hence $\Gamma^{\prime}$ is a normal subgroup of $\Gamma$ of finite index. Then there exists a unramified finite-sheeted covering $\rho_{0}: R^{\prime} \rightarrow R$ such that the fundamental group of $R^{\prime}$ is isomorphic to $\Gamma^{\prime}$ and the covering transformation group of $\rho_{0}: R^{\prime} \rightarrow R$ is isomorphic to $\Gamma / \Gamma^{\prime}$. Let $\pi^{\prime}: M^{\prime} \rightarrow R^{\prime}$ be the fiber product of $\pi: M \rightarrow R$ by $\rho_{0}: R^{\prime} \rightarrow R$, i.e., $M^{\prime}=$ $\left\{\left(p, t^{\prime}\right) \in M \times R^{\prime} \mid \pi(p)=\rho_{0}\left(t^{\prime}\right)\right\}$ and $\pi^{\prime}\left(p, t^{\prime}\right)=t^{\prime}$. Then the fiber $\pi^{\prime-1}\left(t^{\prime}\right)$ of $M^{\prime}$ over $t^{\prime}$ is biholomorphic to the fiber $\pi^{-1}\left(\rho_{0}\left(t^{\prime}\right)\right)$ of $M$ over $\rho_{0}\left(t^{\prime}\right)$, and the monodromy of $\left(M^{\prime}, \pi^{\prime}, R^{\prime}\right)$ with respect to arbitrary $\gamma^{\prime} \in \Gamma^{\prime}$ is $\left[f_{\left(\rho_{0}\right)_{*}\left(\gamma^{\prime}\right)}\right] \in \mathscr{M}^{\prime}$. Since $\tilde{M}$ is biholomorphically equivalent to $\mathbf{B}_{2}$, we see that the universal covering 
space $\tilde{M}^{\prime}$ of $M^{\prime}$ is also biholomorphically equivalent to $\mathbf{B}_{2}$. Therefore we may consider $\left(M^{\prime}, \pi^{\prime}, R^{\prime}\right)$ in place of $(M, \pi, R)$.

5.3. Now suppose that there exists a biholomorphic map $F=\left(F_{1}, F_{2}\right)$ : $\tilde{M} \rightarrow \mathbf{B}_{2}$, and that for every $\Phi_{*}(\gamma)=\left[f_{\gamma}\right] \in \operatorname{Mod}(S), \gamma \in \Gamma$, the quasiconformal self-map $f_{\gamma}: S \rightarrow S$ fixes each puncture of $S$. We may also assume that for every puncture $p_{0}$ of $S$ there exists a neighborhood $U_{p_{0}}$ of $p_{0}$ such that $f_{\gamma}(p)=p$ for all $p \in U_{p_{0}}$.

We set $t_{0}=\rho(0)$, and $S=S_{t_{0}}=\pi^{-1}\left(t_{0}\right) \cong U / G$. Take a cusp point $\zeta_{0}^{*} \in \partial U$ for $G$. From the assumption that the quasiconformal self-map $f_{\gamma}: S \rightarrow S$ inducing $\Phi_{*}(\gamma)$ fixes each puncture of $S$ it follows that for $\Psi_{*}(\gamma)=\left[\omega_{\gamma}\right] \in \operatorname{Mod}(G)$ there exists an element $g_{\gamma} \in G$ such that

$$
g_{\gamma} \circ \omega_{\gamma}(w)=w
$$

for any point $w$ in a cusped region belonging to $\zeta_{0}^{*}$ for $G$.

We set

$$
\begin{aligned}
W^{0}(z) & =\frac{1}{z+1}, \\
G_{0} & =W^{0} G\left(W^{0}\right)^{-1}, \\
\zeta_{0} & =W^{0}\left(\zeta_{0}^{*}\right) \in \partial D_{0}=\partial W^{0}(U) .
\end{aligned}
$$

5.4. Consider the holomorphic motion $V^{\tau}$ of $\partial D_{0}$ given by

$$
V^{\tau}(\zeta)=W^{\mu(\tau)} \circ\left(W^{0}\right)^{-1}(\zeta), \quad(\tau, \zeta) \in \Delta \times \partial D_{0} .
$$

Note that $V$ is $G_{0}$-equivariant, that is, it satisfies the relation

$$
V^{\tau}(g(\zeta))=g^{\tau}\left(V^{\tau}(\zeta)\right) \quad \text { on } \Delta \times \partial D_{0}
$$

for all $g \in G_{0}$, where $g^{\tau}=W^{\mu(\tau)} \circ g \circ\left(W^{\mu(\tau)}\right)^{-1}$. Then an equivariant version of Slodkowski's extension theorem implies that the $G_{0}$-equivariant holomorphic motion $V$ of $\partial D_{0}$ can be extended to a holomorphic motion of $\hat{\mathbf{C}}$ (still called $V^{\tau}$ ) in such a way that (5.2) holds for all $g_{0} \in G_{0}, \tau \in \Delta$, and $w \in \hat{\mathbf{C}}$ (see Earle, Kra and Krushkal' [7], p. 928).

Take a sequence $\left\{w_{n}\right\}_{n=1}^{\infty}$ in a cusped region belonging to $\zeta_{0}$ for $G_{0}$ with $\lim _{n \rightarrow \infty} w_{n}=\zeta_{0}$. We define a holomorphic map $\Delta \rightarrow \tilde{M}$ by

$$
s_{n}(\tau)=\left(\tau, V^{\tau}\left(w_{n}\right)\right),
$$

which is a holomorphic section of $(\tilde{M}, \tilde{\Pi}, \Delta)$. Here $\tilde{\Pi}: \tilde{M} \rightarrow \Delta$ is the holomorphic map given by $\tilde{\Pi}(\tau, w)=\tau$.

We put $h_{\gamma}=\left(\omega_{\gamma}\right)^{-1} \circ g_{\gamma} \circ \omega_{\gamma}$ and

$$
\begin{aligned}
H_{\gamma}(\tau, w) & =H_{\left(\gamma, h_{\gamma}\right)}(\tau, w) \\
& =W^{\mu(\gamma(\tau))} \circ \omega_{\gamma} \circ h_{\gamma} \circ\left(W^{\mu(\tau)}\right)^{-1}(w) .
\end{aligned}
$$


From (5.1) we get

$$
\begin{aligned}
H_{\gamma}\left(\tau, W^{\mu(\tau)}\left(w_{n}\right)\right) & =W^{\mu(\gamma(\tau))} \circ \omega_{\gamma} \circ h_{\gamma} \circ\left(w_{n}\right) \\
& =W^{\mu(\gamma(\tau))} \circ g_{\gamma} \circ \omega_{\gamma}\left(w_{n}\right) \\
& =W^{\mu(\gamma(\tau))}\left(w_{n}\right) .
\end{aligned}
$$

Let $d_{D_{\tau}}$ be the Poincaré distance on $D_{\tau}$. Then we obtain the following lemma:

Lemma 1. There exists a positive constant $K$ depending on $\gamma$ and $\tau$ such that

$$
d_{D_{\gamma}(\tau)}\left(H_{\gamma}\left(\tau, V^{\tau}\left(w_{n}\right)\right), V^{\gamma(\tau)}\left(w_{n}\right)\right) \leq K .
$$

Proof. Noting $H_{\gamma}: D_{\tau} \rightarrow D_{\gamma(\tau)}$ is conformal and (5.3), we get

$$
\begin{aligned}
d_{D_{\gamma}(\tau)} & \left.H_{\gamma}\left(\tau, V^{\tau}\left(w_{n}\right)\right), V^{\gamma(\tau)}\left(w_{n}\right)\right) \\
\leq & d_{D_{\gamma}(\tau)}\left(H_{\gamma}\left(\tau, V^{\tau}\left(w_{n}\right)\right), H_{\gamma}\left(\tau, W^{\mu(\tau)}\left(w_{n}\right)\right)\right) \\
& +d_{D_{\gamma}(\tau)}\left(H_{\gamma}\left(\tau, W^{\mu(\tau)}\left(w_{n}\right)\right), V^{\gamma(\tau)}\left(w_{n}\right)\right) \\
= & d_{D_{\tau}}\left(W^{\mu(\tau)}\left(w_{n}\right), V^{\tau}\left(w_{n}\right)\right)+d_{D_{\gamma}(\tau)}\left(W^{\mu(\gamma(\tau))}\left(w_{n}\right), V^{\gamma(\tau)}\left(w_{n}\right)\right) .
\end{aligned}
$$

Since $V^{\tau}$ is quasiconformal on $\hat{\mathbf{C}}$ by a theorem due to Mañé, Sud and Sullivan (cf. Bers and Royden [6], Theorem 1, p. 492), and $V^{\tau}$ and $W^{\mu(\tau)}$ have the same boundary values on $\partial D_{0}$, Theichmüller's theorem implies that there exists a positive constant $K_{1}$ such that

$$
d_{D_{\tau}}\left(W^{\mu(\tau)}\left(w_{n}\right), V^{\tau}\left(w_{n}\right)\right) \leq K_{1}
$$

for any $n$ (see Theichmüller [24], and Kra [15], Lemma 1, p. 234). Similarly, we find a positive constant $K_{2}$ so that

$$
d_{D_{\gamma}(\tau)}\left(W^{\mu(\gamma(\tau))}\left(w_{n}\right), V^{\gamma(\tau)}\left(w_{n}\right)\right) \leq K_{2}
$$

for any $n$. Hence from (5.5), (5.6) and (5.7) we have

$$
d_{D_{\gamma}(\tau)}\left(H_{\gamma}\left(\tau, V^{\tau}\left(w_{n}\right)\right), V^{\gamma(\tau)}\left(w_{n}\right)\right) \leq K_{1}+K_{2}
$$

for any $n$.

5.5. Since $\mathbf{B}_{2}$ is a bounded domain, we may assume that $\left\{F \circ s_{n}\right\}_{n=0}^{\infty}$ converges uniformly on compact subsets of $\Delta$. We may also assume that

$$
\lim _{n \rightarrow \infty} F \circ s_{n}(\tau)=\lim _{n \rightarrow \infty} F\left(\tau, V^{\tau}\left(w_{n}\right)\right)=(1,0) \in \partial \mathbf{B}_{2}
$$

for every $\tau \in \Delta$ (see Imayoshi [9], pp. 584-585).

Let $F_{*}: \mathscr{G} \rightarrow \operatorname{Aut}\left(\mathbf{B}_{2}\right)$ be the group homomorphism defined by

$$
F \circ(\gamma, g)=F_{*}(\gamma, g) \circ F
$$

for every $(\gamma, g) \in \mathscr{G}=\Gamma \ltimes G$. 
Setting $\chi_{\gamma}=\left(\gamma, h_{\gamma}\right)$, we show that

$$
F_{*}\left(\chi_{\gamma}\right)(1,0)=(1,0)
$$

for all $\gamma \in \Gamma$ as follows. Consider

$$
F \circ \chi_{\gamma}\left(\tau, V^{\tau}\left(w_{n}\right)\right)=F_{*}\left(\chi_{\gamma}\right) \circ F\left(\tau, V^{\tau}\left(w_{n}\right)\right) .
$$

From (5.9) we have

$$
\lim _{n \rightarrow \infty} F_{*}\left(\chi_{\gamma}\right) \circ F\left(\tau, V^{\tau}\left(w_{n}\right)\right)=F_{*}\left(\chi_{\gamma}\right)(1,0) .
$$

Let $d_{\mathbf{B}_{2}}$ be the Kobayashi distance on $\mathbf{B}_{2}$. (For the Kobayashi distance refer to Jarnicki and Pflug [11], and Kobayashi [13].) The distance decreasing property for holomorphic maps with respect to Kobayashi distances guarantees that

$$
\begin{gathered}
d_{\mathbf{B}_{2}}\left(F\left(\gamma(\tau), H_{\gamma}\left(\tau, V^{\tau}\left(w_{n}\right)\right)\right), F\left(\gamma(\tau), V^{\gamma(\tau)}\left(w_{n}\right)\right)\right) \\
\leq d_{D_{\gamma(\tau)}}\left(H_{\gamma}\left(\tau, V^{\tau}\left(w_{n}\right)\right), V^{\gamma(\tau)}\left(w_{n}\right)\right) \leq K .
\end{gathered}
$$

From (5.9) and (5.12) we conclude that

$$
\begin{aligned}
\lim _{n \rightarrow \infty} F \circ \chi_{\gamma}\left(\tau, V^{\tau}\left(w_{n}\right)\right) & =\lim _{n \rightarrow \infty} F\left(\gamma(\tau), H_{\gamma}\left(\tau, V^{\tau}\left(w_{n}\right)\right)\right) \\
& =(1,0) .
\end{aligned}
$$

Therefore form (5.10), (5.11) and (5.13) we have

$$
F_{*}\left(\chi_{\gamma}\right)(1,0)=(1,0)
$$

for any $\gamma \in \Gamma$.

By the same way as Imayoshi [9], pp. 585-587 we can prove Theorem 1 and Corollary 1. This completes the proof of Theorem 1 and Corollary 1 .

\section{Proof of Theorem 2 for $n>0$}

6.1. We recall the following three lemmas:

LEMMA 2. Any analytic automorphism of $\Delta^{2}=(|z|<1) \times(|w|<1)$ is either one of following two types:

(I) $(A, B)(z, w)=(A(z), B(w))$,

(II) $(A, B)(z, w)=(A(w), B(z))$,

where $A, B \in \operatorname{Aut}(\Delta)$.

(See Narashimhan [17], Proposition 3, p. 68.)

Lemma 3. Two Möbius transformations $A$ and $B$ are commutative if and only if $\operatorname{Fix}(A)=\operatorname{Fix}(B)$, i.e, they have the same set of fixed points provided that neither is the identity and provided that $A$ or $B$ is not a transformation of order two.

(See Lehner [16], Theorems 1 and 2, p. 72.) 
Lemma 4. Let $A$ be a hyperbolic or loxodromic transformation and let $B$ be a Möbius transformation which has one and only one fixed point in common with $A$. Then the sequence $\left\{B \circ A^{n} \circ B^{-1} \circ A^{-n}\right\}_{n=1}^{\infty}$ of Möbius transformations converges to a Möbius transformation as $n \rightarrow \infty$ or $-\infty$, so the group $\langle A, B\rangle$ generated by $A$ and $B$ is not discrete.

(See Lehner [16], Theorem 2E, p. 94.)

6.2. Assume that $(M, \pi, R)$ is a holomorphic family of Riemann surfaces of type $(g, n)$ with $n>0$ and there exists a biholomorphic map $F=\left(F_{1}, F_{2}\right)$ : $\tilde{M} \rightarrow \Delta^{2}$.

First assume that for every $\Phi_{*}(\gamma)=\left[f_{\gamma}\right] \in \operatorname{Mod}(S), \gamma \in \Gamma$, the quasiconformal self-map $f_{\gamma}: S \rightarrow S$ fixes every puncture of $S$, and that $F_{*}(\gamma, g)$ is of type (I) for all $(\gamma, g) \in \mathscr{G}=\Gamma \ltimes G$.

We use the notation in $\S 3, \S 4$ and $\S 5$. Let $g_{0}$ be a parabolic element of $G$ with fixed point $\zeta_{0}^{*}$. Set $\zeta_{\tau}=W^{\mu(\tau)}\left(\zeta_{0}^{*}\right) \in \partial D_{\tau}, \tau \in \Delta$. For any $\gamma \in \Gamma$ there exists an element $g_{\gamma} \in G$ satisfying (5.1). We put

$$
\begin{aligned}
h_{\gamma} & =\omega_{\gamma}^{-1} \circ g_{\gamma} \circ \omega_{\gamma}, \\
H_{\gamma}\left(\tau, \zeta_{\tau}\right) & =H_{\left(\gamma, h_{\gamma}\right)}\left(\tau, \zeta_{\tau}\right)=W^{\mu(\gamma(\tau))} \circ\left(\omega_{\gamma} \circ h_{\gamma}\right) \circ\left(W^{\mu(\tau)}\right)^{-1}\left(\zeta_{\tau}\right) .
\end{aligned}
$$

Then we obtain

$$
\zeta_{\gamma(\tau)}=H_{\gamma}\left(\tau, \zeta_{\tau}\right)
$$

We put

$$
\left(A_{\gamma}, B_{\gamma}\right) \circ F=F \circ\left(\gamma, h_{\gamma}\right)
$$

where $\left(A_{\gamma}, B_{\gamma}\right) \in \operatorname{Aut}\left(\Delta^{2}\right)$.

Using the holomorphic motion $V^{\tau}$ in $\S 5.4$, we define a sequence $\left\{\left(\varphi_{n}, \psi_{n}\right)\right\}_{n=1}^{\infty}$ of holomorphic maps from $\Delta$ into $\Delta^{2}$ by

$$
\left(\varphi_{n}(\tau), \psi_{n}(\tau)\right)=\left(F_{1}\left(\tau, V^{\tau}\left(w_{n}\right)\right), F_{2}\left(\tau, V^{\tau}\left(w_{n}\right)\right)\right) .
$$

We may assume that $\left\{\left(\varphi_{n}, \psi_{n}\right)\right\}_{n=1}^{\infty}$ converges uniformly to a holomorphic map $\left(\varphi_{0}, \psi_{0}\right): \Delta \rightarrow \bar{\Delta}^{2}$ on compact subsets of $\Delta$. Then the maximum theorem for holomorphic functions yields one of the following four cases:

(1) $\left(\varphi_{0}, \psi_{0}\right)(\Delta) \subset \Delta^{2}$.

(2) $\left(\varphi_{0}, \psi_{0}\right)$ is constant on $\Delta$ with value $\left(c_{1}, c_{2}\right) \in(\partial \Delta)^{2}$.

(3) $\varphi_{0}$ is constant on $\Delta$ with value $c_{1} \in \partial \Delta$, and $\psi_{0}(\Delta) \subset \Delta$.

(4) $\varphi_{0}(\Delta) \subset \Delta$, and $\psi_{0}$ is constant on $\Delta$ with value $c_{2} \in \partial \Delta$.

Since $F$ is a proper map, case (1) does not occur. We show that case (2) neither occurs as follows. Assume that $\left(\varphi_{0}, \psi_{0}\right)$ is constant on $\Delta$ with value $\left(c_{1}, c_{2}\right) \in(\partial \Delta)^{2}$. From (5.8), (6.1), (6.2) and (6.3), for any $\gamma \in \Gamma$ we obtain

$$
A_{\gamma}\left(c_{1}\right)=c_{1}, \quad \text { and } \quad B_{\gamma}\left(c_{2}\right)=c_{2} \text {. }
$$


Take two elements $\gamma, \delta \in \Gamma$ with $\gamma \delta \neq \delta \gamma$. Then we have $\left(\gamma, h_{\gamma}\right) \circ\left(\delta, h_{\delta}\right) \neq\left(\delta, h_{\delta}\right) \circ$ $\left(\gamma, h_{\gamma}\right)$, and so $\left(A_{\gamma}, B_{\gamma}\right) \circ\left(A_{\delta}, B_{\delta}\right) \neq\left(A_{\delta}, B_{\delta}\right) \circ\left(A_{\gamma}, B_{\gamma}\right)$. Hence we get

$$
A_{\gamma} A_{\delta} \neq A_{\delta} A_{\gamma}, \quad \text { or } \quad B_{\gamma} B_{\delta} \neq B_{\delta} B_{\gamma} .
$$

Therefore Lemmas $3,4,(6.4)$ and (6.5) imply that $F_{*}(\mathscr{G})$ is not discrete. This is a contradiction.

In case (3) we see that $\psi_{0}$ is not constant as follows. Suppose that $\psi_{0}$ is constant with value $c_{2} \in \Delta$. From (5.8), (6.1), (6.2) and (6.3), for any $\gamma \in \Gamma$ we have

$$
A_{\gamma}\left(c_{1}\right)=c_{1}, \quad \text { and } \quad B_{\gamma}\left(c_{2}\right)=c_{2} \text {. }
$$

Let $c_{1}^{*}$ be the reflection of $c_{1}$ with respect to the unit circle $\partial \Delta$. Since $A_{\gamma} \in \operatorname{Aut}(\Delta)$, we see that

$$
A_{\gamma}\left(c_{1}^{*}\right)=c_{1}^{*}
$$

for any $\gamma \in \Gamma$. Hence Lemma 3, (6.6) and (6.7) imply that

$$
A_{\gamma} A_{\delta}=A_{\delta} A_{\gamma}
$$

for all $\gamma, \delta \in \Gamma$.

Take two elements $\gamma, \delta \in \Gamma$ with $\gamma \delta \neq \delta \gamma$. Then we have $\left(\gamma, h_{\gamma}\right) \circ\left(\delta, h_{\delta}\right) \neq$ $\left(\delta, h_{\delta}\right) \circ\left(\gamma, h_{\gamma}\right)$, and so $\left(A_{\gamma}, B_{\gamma}\right) \circ\left(A_{\delta}, B_{\delta}\right) \neq\left(A_{\delta}, B_{\delta}\right) \circ\left(A_{\gamma}, B_{\gamma}\right)$. Noting (6.8) we get

$$
B_{\gamma} B_{\delta} \neq B_{\delta} B_{\gamma} .
$$

Therefore Lemmas 3, 4, (6.8) and (6.9) imply that $F_{*}(\mathscr{G})$ is not discrete. This is a contradiction.

Now assume that $\varphi_{0}$ is constant on $\Delta$ with value $c_{1} \in \partial \Delta$ and $\psi_{0}: \Delta \rightarrow \Delta$ is a non-constant holomorphic map. Let $F \circ\left(1, g_{0}\right)=\left(A_{0}, B_{0}\right) \circ F$. Then from (6.1), (6.2) and (6.3) we obtain

$$
\varphi_{0}(\tau)=A_{0} \circ \varphi_{0}(\tau)=A_{0}\left(c_{1}\right)=c_{1}, \quad \text { and } \quad \psi_{0}(\tau)=B_{0} \circ \psi_{0}(\tau) .
$$

Since $\psi_{0}$ is not constant, we see that $A_{0}\left(c_{1}\right)=c_{1}$, and $B_{0}=1$, and so $F_{*}\left(1, g_{0}\right)=$ $\left(A_{0}, 1\right)$, where $A_{0}$ is of infinite order and has a fixed point $c_{1} \in \partial \Delta$. By a theorem due to Shimizu [23] (Theorem 2, p. 39), we see that

$$
\begin{aligned}
& \mathscr{G}_{1}^{*}=\left\{A_{g} \in \operatorname{Aut}(\Delta) \mid\left(A_{g}, B_{g}\right)=F_{*}(1, g), g \in G\right\}, \\
& \mathscr{G}_{2}^{*}=\left\{B_{g} \in \operatorname{Aut}(\Delta) \mid\left(A_{g}, B_{g}\right)=F_{*}(1, g), g \in G\right\}
\end{aligned}
$$

are discrete.

If $\left.F_{2}\right|_{D_{\tau}}: D_{\tau} \rightarrow \Delta$ is not constant, then $\left.F_{2}\right|_{D_{\tau}}$ induces a non-constant holomorphic map $\left[F_{2}\right]_{\tau}: D_{\tau} / G_{\tau} \rightarrow \Delta / \mathscr{G}_{2}^{*}$. Since the Riemann surface $D_{\tau} / G_{\tau}$ is of analytically finite type, we see that $\left[F_{2}\right]_{\tau}$ has a holomorphic extension to the compactification of $D_{\tau} / G_{\tau}$. Hence we have $\psi_{0}(\tau) \in \partial \Delta$, and so by the maximum principle we conclude that $\psi_{0}$ is constant on $\Delta$, which is a contradiction. Therefore, $F_{2}$ is constant on $D_{\tau}$ for all $\tau \in \Delta$, and $F_{*}(1, g)$ is of form $\left(A_{g}, 1\right)$ for any $g \in G$, i.e., 


$$
\mathscr{G}_{1}^{*}=\left\{A_{g} \in \operatorname{Aut}(\Delta) \mid\left(A_{g}, 1\right)=F_{*}(1, g), g \in G\right\} .
$$

Therefore $S_{\rho(\tau)} \cong D_{\tau} / G_{\tau} \cong \Delta / \mathscr{G}_{1}^{*}$ for every $\tau \in \Delta$. Similarly, in case (4) we can show that all fibers $S_{t}$ are biholomorphically equivalent.

6.3. Next we prove that $F_{*}(\gamma, g)$ is of type (I) for all $(\gamma, g) \in \mathscr{G}=\Gamma \ltimes G$ provided that for every $\Phi_{*}(\gamma)=\left[f_{\gamma}\right] \in \operatorname{Mod}(S), \gamma \in \Gamma$, the quasiconformal selfmap $f_{\gamma}: S \rightarrow S$ fixes every puncture of $S$. Assume that $\mathscr{G}_{0}^{*}=\{(A, B) \mid(A, B)=$ $F \circ(\gamma, g) \circ F^{-1}$ is of type $\left.(\mathrm{I}),(\gamma, g) \in \Gamma \ltimes G\right\}$ is a subgroup of $\mathscr{G}^{*}$ of index two. By the same argument as in $\$ 6.2$ we see that one of the following two cases holds:

(1) $F_{1}$ is constant and $F_{2}$ is non-constant on $D_{\tau}$ for all $\tau \in \Delta$.

(2) $F_{2}$ is constant and $F_{1}$ is non-constant on $D_{\tau}$ for all $\tau \in \Delta$.

In case (1), if $(A, B)=(A, B)=F \circ(\gamma, g) \circ F^{-1}$ is of type (II) for some $(\gamma, g)$, then we have $F_{1}\left(\gamma(\tau), H_{(\gamma, g)}(\tau, w)\right)=A \circ F_{2}(\tau, w)$. Since $F_{1}$ is constant and $A \circ F_{2}$ is non-constant on $D_{\tau}$, we have a contradiction. Hence every $F \circ(\gamma, g) \circ F^{-1}$ is of type (I). Similarly it follows that in case (2), every $F \circ(\gamma, g) \circ F^{-1}$ is of type (I).

6.4. If $\Gamma$ has an element $\gamma$ such that $\Phi_{*}(\gamma)=\left[f_{\gamma}\right] \in \operatorname{Mod}(S)$ does not fix a puncture of $S$, then the same reasoning as one in $\$ 5.2$ implies that all fibers $S_{t}$ are biholomorphically equivalent.

\section{Proof of Theorem 2 for a compact complex surface $M$}

7.1. We shall give a proof of Theorem 2 in the case where $M$ is compact, that is, the base surface $R$ is compact and $n=0$, i.e., every fiber $S_{t}=\pi^{-1}(t)$ is also compact.

Assume that there exists a biholomorphic map $F=\left(F_{1}, F_{2}\right): \Delta^{2} \rightarrow \tilde{M}$. We also assume that every element of $\mathscr{G}^{*}=F^{-1} \mathscr{G} F$ is of type (I).

We shall show that $F=\left(F_{1}, F_{2}\right)$ satisfies the following:

$$
\frac{\partial F_{1}}{\partial z}=0 \quad \text { on } \Delta^{2}, \quad \text { or } \quad \frac{\partial F_{1}}{\partial w}=0 \quad \text { on } \Delta^{2} .
$$

In order to obtain (7.1) we show that

$$
\lim _{n \rightarrow \infty} \frac{\partial F_{1}}{\partial w}\left(z_{n}, w_{n}\right) \times \frac{\partial F_{2}}{\partial w}\left(z_{n}, w_{n}\right)=0
$$

for any point $\left(\zeta_{0}, w_{0}\right) \in \partial \Delta \times \Delta$ and any sequence $\left\{\left(z_{n}, w_{n}\right)\right\}_{n=1}^{\infty}$ of points in $\Delta^{2}$ with $\lim _{n \rightarrow \infty}\left(z_{n}, w_{n}\right)=\left(\zeta_{0}, w_{0}\right)$.

Suppose that (7.2) does not hold for some $\left(\zeta_{0}, w_{0}\right)$ and $\left\{\left(z_{n}, w_{n}\right)\right\}_{n=1}^{\infty}$. Then there exists a positive constant $\varepsilon_{0}$ and a subsequence $\left\{\left(z_{n_{j}}, w_{n_{j}}\right)\right\}_{j=1}^{\infty}$ such that

$$
\left|\frac{\partial F_{1}}{\partial w}\left(z_{n_{j}}, w_{n_{j}}\right) \times \frac{\partial F_{2}}{\partial w}\left(z_{n_{j}}, w_{n_{j}}\right)\right| \geq \varepsilon_{0}
$$

for all $j$. 
Since $\tilde{M}$ is a bounded domain, we may assume that the sequence $\left\{F\left(z_{n_{j}}, \cdot\right)\right\}_{j=1}^{\infty}$ of holomorphic maps $F\left(z_{n_{j}}, \cdot\right): \Delta=(|w|<1) \rightarrow \tilde{M}$ converges to a holomorphic map $\varphi=\left(\varphi_{1}, \varphi_{2}\right): \Delta \rightarrow \overline{\tilde{M}}$ uniformly on compact subsets of $\Delta$, where $\overline{\tilde{M}}$ is the closure of $\tilde{M}$.

Let $\mathscr{F}$ be a fundamental set for $\mathscr{G}^{*}$. Note that $\mathscr{F} \Subset \Delta^{2}$, for $M$ is compact. Then we can find a sequence $\left\{\left(a_{j}, b_{j}\right)\right\}_{j=1}^{\infty}$ of points in $\mathscr{F}$ and a sequence $\left\{A_{j}, B_{j}\right\}_{j=1}^{\infty}$ of elements in $\mathscr{G}^{*}$ such that

$$
\left(A_{j}, B_{j}\right)\left(a_{j}, b_{j}\right)=\left(A_{j}\left(a_{j}\right), B_{j}\left(b_{j}\right)\right)=\left(z_{n_{j}}, w_{n_{j}}\right) .
$$

We may assume that $\left(a_{j}, b_{j}\right)$ converges to a point $\left(a_{0}, b_{0}\right) \in \Delta^{2}$. We may also assume that $\left(A_{j}, B_{j}\right)$ converges to $\left(\zeta_{0}, B_{0}\right)$ uniformly on compact subsets of $\Delta^{2}$, where $\zeta_{0}$ is the constant map with value $\zeta_{0}$ and $B_{0} \in \operatorname{Aut}(\Delta)$. Because conditions $\lim _{j \rightarrow \infty} a_{j}=a_{0} \in \Delta$ and $\lim _{j \rightarrow \infty} A_{j}\left(a_{j}\right)=\zeta_{0} \in \partial \Delta$ imply $\lim _{j \rightarrow \infty} A_{j}=\zeta_{0}$, and conditions $\lim _{j \rightarrow \infty} b_{j}=b_{0} \in \Delta$ and $\lim _{j \rightarrow \infty} B_{j}\left(b_{j}\right)=w_{0} \in \Delta$ imply $\lim _{j \rightarrow \infty} B_{j}=$ $B_{0} \in \operatorname{Aut}(\Delta)$.

We put $F_{*}\left(A_{j}, B_{j}\right)=F \circ\left(A_{j}, B_{j}\right) \circ F^{-1}=\left(\gamma_{j}, g_{j}\right) \in \mathscr{G}=\Gamma \ltimes G . \quad$ Then we have

$$
\begin{aligned}
& F_{1}\left(A_{j}\left(a_{j}\right), B_{j}\left(b_{j}\right)\right)=\gamma_{j} \circ F_{1}\left(a_{j}, b_{j}\right), \\
& F_{2}\left(A_{j}\left(a_{j}\right), B_{j}\left(b_{j}\right)\right)=H_{j}\left(F_{1}\left(a_{j}, b_{j}\right), F_{2}\left(a_{j}, b_{j}\right)\right),
\end{aligned}
$$

where $H_{j}=H_{\left(\gamma_{j}, g_{j}\right)}$.

Since $F: \Delta^{2} \rightarrow \tilde{M}$ is biholomorphic, we see that $\left(\gamma_{j}, g_{j}\right) \circ F\left(a_{j}, b_{j}\right)=$ $F\left(z_{n_{j}}, w_{n_{j}}\right)$ converges to a boundary point $\varphi\left(w_{0}\right)=\left(\varphi_{1}\left(w_{0}\right), \varphi_{2}\left(w_{0}\right)\right)$ of $\tilde{M}$.

If $\varphi_{1}\left(w_{0}\right) \in \partial \Delta=(|\tau|<1)$, then we may assume that $\left\{\gamma_{j}\right\}_{j=1}^{\infty}$ converges to a constant map $\varphi_{1}\left(w_{0}\right)$ uniformly on compact subsets of $\Delta$, because $\gamma_{j} \in \operatorname{Aut}(\Delta)$, $\lim _{j \rightarrow \infty} F_{1}\left(a_{j}, b_{j}\right)=F_{1}\left(a_{0}, b_{0}\right) \in \Delta$, and $\lim _{j \rightarrow \infty} \gamma_{j} \circ F_{1}\left(a_{j}, b_{j}\right)=\lim _{j \rightarrow \infty} F_{1}\left(z_{n_{j}}, b_{n_{j}}\right)$ $=\varphi_{1}\left(w_{0}\right) \in \partial \Delta$. Hence from

$$
\begin{aligned}
\frac{d \gamma_{j} \circ F_{1}\left(a_{j}, w\right)}{d w} & =\frac{d F_{1}\left(A_{j}\left(a_{j}\right), B_{j}(w)\right)}{d w} \\
& =\frac{d F_{1}\left(z_{n_{j}}, B_{j}(w)\right)}{d w} \\
& =\frac{\partial F_{1}}{\partial w}\left(z_{n_{j}}, B_{j}(w)\right) \times B_{j}^{\prime}(w)
\end{aligned}
$$

we obtain

$$
\lim _{j \rightarrow \infty} \frac{\partial F_{1}}{\partial w}\left(z_{n_{j}}, B_{j}(w)\right) \times B_{0}^{\prime}(w)=0 .
$$

Since $B_{j}\left(b_{j}\right)=w_{n_{j}}$ and $B_{0}^{\prime}(w) \neq 0$, we conclude that

$$
\lim _{j \rightarrow \infty} \frac{\partial F_{1}}{\partial w}\left(z_{n_{j}}, w_{n_{j}}\right)=0 .
$$


Since $\lim _{j \rightarrow \infty} \partial F_{2} / \partial w\left(z_{n_{j}}, w_{n_{j}}\right)$ exists, we have

$$
\lim _{j \rightarrow \infty} \frac{\partial F_{1}}{\partial w}\left(z_{n_{j}}, w_{n_{j}}\right) \times \frac{\partial F_{2}}{\partial w}\left(z_{n_{j}}, w_{n_{j}}\right)=0,
$$

which is a contradiction to (7.3).

If $\varphi_{1}\left(w_{0}\right) \in \Delta=(|\tau|<1)$, then we may assume that there exists an element $\gamma_{0} \in \Gamma$ such that $\gamma_{j}=\gamma_{0}$ for any $j$. In fact, assuming $\gamma_{j}$ converges to a holomorphic map $\gamma_{0}: \Delta \rightarrow \bar{\Delta}$ uniformly on compact subsets of $\Delta$, the assumptions $\lim _{j \rightarrow \infty} F_{1}\left(a_{j}, b_{j}\right)=F_{1}\left(a_{0}, b_{0}\right) \in \Delta$ and $\lim _{j \rightarrow \infty} \gamma_{j} \circ F_{1}\left(a_{j}, b_{j}\right) \rightarrow \varphi\left(w_{0}\right) \in \Delta$ imply that $\gamma_{0} \in \operatorname{Aut}(\Delta)$, and the discreteness of $\Gamma$ implies that $\gamma_{j}=\gamma_{k}$ for all sufficiently large $j$ and $k$. Let $\tau_{j}=F_{1}\left(a_{j}, b_{j}\right)$ and $\tau_{0}=F_{1}\left(a_{0}, b_{0}\right)$. Then $H_{j}\left(\tau_{j}, \cdot\right): D_{\tau_{j}} \rightarrow$ $D_{\gamma_{0}\left(\tau_{j}\right)}$ is conformal and we may assume that $\left\{H_{j}\left(\tau_{j}, \cdot\right)\right\}_{j=1}^{\infty}$ converges to a holomorphic map $H_{0}: D_{\tau_{0}} \rightarrow \overline{D_{\gamma_{0}}\left(\tau_{0}\right)}$ uniformly on compact subsets of $D_{\tau_{0}}$. Since $H_{0}\left(F\left(a_{0}, b_{0}\right)\right)=\varphi_{2}\left(w_{0}\right) \in \partial D_{\gamma_{0}\left(\tau_{0}\right)}$, we see that $H_{0}$ is constant on $D_{\tau_{0}}$. Hence from

$$
\begin{aligned}
\frac{d H_{j} \circ F\left(a_{j}, w\right)}{d w} & =\frac{d F_{2}\left(A_{j}\left(a_{j}\right), B_{j}(w)\right)}{d w} \\
& =\frac{d F_{2}\left(z_{n_{j}}, B_{j}(w)\right)}{d w} \\
& =\frac{\partial F_{2}}{\partial w}\left(z_{n_{j}}, B_{j}(w)\right) \times B_{j}^{\prime}(w)
\end{aligned}
$$

we obtain

$$
\lim _{j \rightarrow \infty} \frac{\partial F_{2}}{\partial w}\left(z_{n_{j}}, B_{j}(w)\right) \times B_{0}^{\prime}(w)=0 .
$$

Since $B_{j}\left(b_{j}\right)=w_{n_{j}}$ and $B_{0}^{\prime}(w) \neq 0$, we conclude that

$$
\lim _{j \rightarrow \infty} \frac{\partial F_{2}}{\partial w}\left(z_{n_{j}}, w_{n_{j}}\right)=0 .
$$

Since $\lim _{j \rightarrow \infty} \partial F_{1} / \partial w\left(z_{n_{j}}, w_{n_{j}}\right)$ exists, we have

$$
\lim _{j \rightarrow \infty} \frac{\partial F_{1}}{\partial w}\left(z_{n_{j}}, w_{n_{j}}\right) \times \frac{\partial F_{2}}{\partial w}\left(z_{n_{j}}, w_{n_{j}}\right)=0,
$$

which is a contradiction to (7.3).

Therefore we have (7.2) for any point $\left(\zeta_{0}, w_{0}\right) \in \partial \Delta \times \Delta$ and any sequence $\left\{\left(z_{n}, w_{n}\right)\right\}_{n=1}^{\infty}$ of points in $\Delta^{2}$ with $\lim _{n \rightarrow \infty}\left(z_{n}, w_{n}\right)=\left(\zeta_{0}, w_{0}\right)$. Then Radó's theorem implies

$$
\frac{\partial F_{1}}{\partial w} \times \frac{\partial F_{2}}{\partial w}=0 \text { on } \Delta^{2} .
$$

(See Narashimhan [17], Theorem 1, p. 53). Hence we have

$$
\frac{\partial F_{1}}{\partial w}=0 \quad \text { on } \Delta^{2}, \quad \text { or } \quad \frac{\partial F_{2}}{\partial w}=0 \text { on } \Delta^{2} .
$$


By a similar way as above we obtain

$$
\frac{\partial F_{1}}{\partial z}=0 \quad \text { on } \Delta^{2}, \quad \text { or } \quad \frac{\partial F_{2}}{\partial z}=0 \quad \text { on } \Delta^{2} .
$$

Since

$$
\operatorname{det}\left(\begin{array}{ll}
\frac{\partial F_{1}}{\partial z} & \frac{\partial F_{1}}{\partial w} \\
\frac{\partial F_{2}}{\partial z} & \frac{\partial F_{2}}{\partial w}
\end{array}\right)
$$

does not vanish at every point of $\Delta^{2}$, from (7.7) and (7.8) we see that one of the following two relations holds:

(i) $\partial F_{1} / \partial z=\partial F_{2} / \partial w=0$ on $\Delta^{2}$,

(ii) $\partial F_{1} / \partial w=\partial F_{2} / \partial z=0$ on $\Delta^{2}$.

If relation (i) holds, then $F_{1}(z, w)=F_{1}(w)$, i.e., $F_{1}$ is independent on $z$. Then $F^{-1} \circ(1, g) \circ F$ is of form $\left(A_{g}, 1\right)$ and of type (I) for every $g \in G$. Thus setting $\mathscr{A}_{G}^{*}=\left\{A_{g} \mid\left(A_{g}, 1\right)=F^{-1} \circ(1, g) \circ F, g \in G\right\}$, we see that

$$
S_{\rho(\tau)} \cong D_{\tau} / G_{\tau} \cong \Delta / \mathscr{A}_{G}^{*}
$$

for any $\tau \in \Delta$, which concludes that all the fibers $S_{t}$ are biholomorphically equivalent.

If relation (ii) holds, then $F_{1}(z, w)=F_{1}(z)$, and $F^{-1} \circ(1, g) \circ F$ is of form $\left(1, B_{g}\right)$ and of type $(\mathrm{I})$ for every $g \in G$. Thus we have

$$
S_{\rho(\tau)} \cong D_{\tau} / G_{\tau} \cong \Delta / \mathscr{B}_{G}^{*}
$$

for any $\tau \in \Delta$, where $\mathscr{B}_{G}^{*}=\left\{B_{g} \mid\left(1, B_{g}\right)=F^{-1} \circ(1, g) \circ F, g \in G\right\}$. Hence all the fibers $S_{t}$ are biholomorphically equivalent.

7.2. Let $M$ be compact, and assume that there exists a biholomorphic map $F=\left(F_{1}, F_{2}\right): \Delta^{2} \rightarrow \tilde{M}$, and $\mathscr{G}^{*}=F^{-1} \mathscr{G} F$ has an element of type (II). Let $\mathscr{G}_{0}^{*}$ be the set all elements of type (I) in $\mathscr{G}^{*}$, which is a normal subgroup of $\mathscr{G}^{*}$ of index two. Using $\mathscr{G}_{0}^{*}$ in place of $\mathscr{G}^{*}$, the same way as in $\$ 7.1$ we see that $F_{1}(z, w)=F_{1}(w)$ or $F_{1}(z, w)=F_{1}(z)$. If $F_{1}(z, w)=F_{1}(w)$, then $F^{-1} \circ(1, g) \circ F$ is of form $\left(A_{g}, 1\right)$ and of type (I) for every $g \in G$. Hence by the same reasoning as above we obtain

$$
S_{\rho(\tau)} \cong D_{\tau} / G_{\tau} \cong \Delta / \mathscr{A}_{G}^{*}
$$

for any $\tau \in \Delta$, which concludes that all the fibers $S_{t}$ are biholomorphically equivalent.

Similarly if $F_{1}(z, w)=F_{1}(z)$, then we see that

$$
S_{\rho(\tau)} \cong D_{\tau} / G_{\tau} \cong \Delta / \mathscr{B}_{G}^{*} .
$$

Hence all the fibers $S_{t}$ are biholomorphically equivalent.

This completes the proof of Theorem 2 in the case where $M$ is compact. 
7.3. In the case where the base surface $R$ is not compact, a proof of Theorem 2 is given Imayoshi [9], pp. 587-596.

If all the fibers $S_{t}=\pi^{-1}(t)$ are biholomorphically equivalent, then the representation $\Psi$ of $(M, \pi, R)$ into $T(G)$ is constant, and so $\tilde{M}=\Delta \times D_{0} \cong(\Delta)^{2}$. This completes the proof of Theorem 2 .

Finally we note that a proof of Corollary 2 is given in Imayoshi [9], p. 587.

\section{REFERENCES}

[1] M. F. Атіуaн, The signature of fibre-bundles, in "Global Analysis, Papers in honor of K. Kodaira”, edited by D. C. Spencer and S. Iyanaga, University of Tokyo Press and Princeton University Press, 1969, 73-84.

[2] W. Barth, C. Peters and A. Van de Ven, Compact Complex Surfaces, Springer-Verlag, Berlin, Heidelberg and New York, 1984.

[ 3 ] L. BERs, Fiber spaces over Teichmüller spaces, Acta. Math. 130 (1973), 89-126.

[4] L. Bers, On Hilbert's 22nd problem, Proc. Sympos. Pure. Math. 28 (1976), 559-609.

[5] L. BERs, Finite dimensional Teichmüller spaces and generalizations, Bull. of Amer. Math. Soc. (New Series) 5 (1981), 131-172.

[6] L. Bers AND H. L. Royden, Holomorphic families of injections, Acta. Math. 157 (1986), 259-286.

[7] C. J. Earle, I. Kra, and S. L. KrushKal', Holomorphic motions and Teichmüller spaces, Trans. of Amer. Math. Soc. 343 (1994), 927-948.

[ 8 ] P. A. Griffiths, Complex analytic properties of certain Zariski open sets on algebraic varieties, Ann. of Math. 94 (1971), 21-55.

[9] Y. Imayoshi, Universal covering spaces of certain quasi-projective algebraic surfaces, Osaka J. Math. 20 (1983), 581-598.

[10] Y. Imayoshi and M. Taniguchi, An Introduction to Teichmüller Spaces, Springer-Verlag, Tokyo and New York, 1992.

[11] M. Jarnicki And P. Pflug, Invariant Distances and Metrics in Complex Analysis, Walter de Gruyter, Berlin and New York, 1993.

[12] A. KAS, On deformations of a certain type of irregular algebraic surface, Amer. J. Math. 90 (1968), 789-804.

[13] S. KоваYashi, Hyperbolic Complex Spaces, Springer-Verlag, Berlin, Heidelberg and New York, 1998.

[14] K. Kodaira, A certain type of irregular algebraic surfaces, J. d'Analyse Math. 19 (1967), 207-215.

[15] I. KRA, On the Nielsen-Thurston-Bers type of some self-maps of Riemann surfaces, Acta Math. 146 (1981), 231-270.

[16] J. LeHner, Discontinuous groups and automorphic functions, American Mathematical Society, Rohde Island, 1964.

[17] R. Narasimhan, Several complex variables, University of Chicago Press, Chicago, 1971.

[18] G. RIERA, Semi-direct products of Fuhsian groups and uniformization, Duke Math. J. 44 (1977), 291-304.

[19] J. P. Rosay, Sur une caractérisation de la boule parmi les domaines de $\mathbf{C}^{n}$ par son groupe d'automorphismes, Ann. Inst. Fourier 29-4 (1979), 91-97.

[20] G. B. Shabat, The complex structure of domains covering algebraic surfaces, Functional Anal. Appl. 11 (1976), 135-142.

[21] G. B. Shabat, Local construction of complex algebraic surfaces with respect to the universal covering, Functional Anal. Appl. 17 (1983), 157-159. 
UNIVERSAL COVERINGS OF HOLOMORPHIC FAMILIES OF RIEMANN SURFACES 247

[22] I. R. Shafarevich, Basic Algebraic Geometry, Springer-Verlag, Berlin, Heidelberg and New York, 1974.

[23] H. Shimizu, On discontinuous groups operating on the product of the upper half planes, Ann. of Math. 77 (1963), 33-71.

[24] O. TeichmüLlER, Ein Verschiebungssatz der quasikonformen Abbildung, Deutsche Math. 7 (1944), 336-343.

\author{
YoICHI IMAYOSHI \\ Department of Mathematics \\ OSAKa City UNIVERSITY \\ Sugimoto, Sumiyoshi-Ku, Osaka 558-0022 \\ JAPAN \\ E-mail address: imayoshi@sci.osaka-cu.ac.jp \\ MinORI Nishimura \\ Department of Mathematics \\ Osaka City University \\ Sugimoto, Sumiyoshi-Ku, OsaKa 558-0022 \\ JAPAN \\ E-mail address: minori@sci.osaka-cu.ac.jp
}

